\title{
Treatment of Human Responses
}

\author{
Moderator: \\ Duane Pennebaker, R.N., Ph.D. \\ Assistant Professor/Director \\ Graduate Program, School of Nursing \\ University of Alaska, Anchorage \\ Anchorage, Alaska
}

Laurel Ann Steinhaus

Family Nurse Practitioner

Hospital Based Home Care (111J)

Veterans Administration Hospital

Salt Lake City, Utah

Joy C. Miller

Graduate Student

Department of Physiological Nursing

University of Washington

Seattle, Washington

Margaret M. Heitkemper

Asssistant Professor

Department of Physiological Nursing

University of Washington

Seattle, Washington

Eleanor L. Strang

Instructor

College of Nursing

Arizona State University

Tempe, Arizona
Cardiovascular Effects of an Aerobic Walking Program on Older Adults

Effect of Restricted Liquid Feeding on Gastrointestinal Parameters and Plasma Corticosterone Rhythmicity in Rats

Effect of Topical Lidocaine During Painful Wound Debridement 
Marian Romero

Instructor (formerly)

School of Nursing

The University of Michigan

Ann Arbor, Michigan

Marie Neaton

Instructor

School of Nursing

The University of Michigan

Ann Arbor, Michigan

Discussant

Ann J. Davis

Professor

Mental Health \& Community Nursing

University of California

San Francisco
Ethics of Clinical Intervention Studies 


\title{
Cardiovascular Effects of an Aerobic Walking Program in Older Adults
}

\author{
Laurel Ann Steinhaus, M.S., F.N.C. \\ Family Nurse Practitioner \\ Hospital Based Home Care (111J) \\ Veterans Administration Hospital \\ Salt Lake City, Utah
}

Twenty-eight healthy, sedentary 55-70 year-old men and women participated in a study designed to determine the effects of aerobic exercise on the cardiovascular system. An experimental design with repeated measures was used. Dependent variables studied during a pre and posttreatment maximal exercise treadmill test were: heart rate, blood pressure, maximal oxygen consumption and physical work capacity. The experimental group $(N=13)$ participated in four months of supervised aerobic walking exercise for three, one hour sessions per week while the control group received nonaerobic stretching exercises. Gains in maximal oxygen consumption were significantly different between groups $(t=1.19, p<.006)$, with the experimental and control groups had significant pre-post improvement in maximal oxygen consumption, maximal work rate and time on the treadmill $(p<.05)$. A decrease in resting heart rate and systolic blood pressure at rest was noted in both groups. A post test questionnaire revealed self-reported improvement in sleep habits, dietary patterns, reduction in the use of medications, and an increased feeling of wellbeing following both programs. Stretching exercises as well as aerobics exercises induced beneficial hemodynamic and lifestyle changes in these older adults.

This project was supported by the Veterans Administration and by funds from NIH Biomedical Research Support (Grant \#4407092) administered through the Neuropsychology Department. 


\title{
Effect of Restricted Liquid Feeding on Gastrointestinal Parameters and Plasma Corticosterone Rhythmicity in Rats
}

\author{
Joy C. Miller, R.N., M.N. \\ Graduate Student \\ Department of Physiological Nursing \\ University of Washington \\ Seattle, Washington \\ Margaret M. Heitkemper, R.N., Ph.D. \\ Assistant Professor \\ Department of Physiological Nursing \\ University of Washington \\ Seattle, Washington
}

Physiological adaptation to enteral feedings may be dependent on nutrient and bulk content of the diet as well as method of administration including time of delivery. This study investigated the influence of liquid diet and restricted feeding on body weight, gastrointestinal parameters, and plasma corticosterone rhythmicity in rats.

Thirty-six male Sprague-Dawley rats were divided into three groups: Group 1 received rat chow ad libitum, Group 2 received a liquid diet ad libitum, Group 3 was restricted to a liquid diet between $0600-1800 \mathrm{hr}$. Following two weeks on these feeding regimens, three rats from each group were sacrificed at 0600 , 1200, 1800, 2400hr.

Significant $(p<0.05)$ decreases in body weight were found in Group 2 and 3 rats as compared with Group 1. Animals on the liquid diet also had differences in small intestine weight and protein content as compared to rats receiving the solid diet. Groups 1 and 2 demonstrated normal circadian rhythmicity in plasma corticosterone levels while Group 3 exhibited a $12 \mathrm{hr}$. shift in plasma corticosterone levels.

The present study supports the interrelationship between timing of food presentation and diurnal rhythmicity of plasma corticosterone levels, liver weights, and protein contents. Restricted feeding may significantly alter normal biological rhythms. Clinical studies of enteral feeding schedules are warranted. 


\title{
Effect of Topical Lidocaine During \\ Painful Wound Debridement
}

\author{
Eleanor L. Strang, R.N., M.S. \\ Instructor \\ College of Nursing \\ Arizona State University \\ Tempe, Arizona \\ Marian Romero, R.N., M.N. \\ Instructor (formerly) \\ School of Nursing \\ The University of Michigan \\ Ann Arbor, Michigan \\ Marie Neaton, R.N., M.S. \\ Instructor \\ School of Nursing \\ The University of Michigan \\ Ann Arbor, Michigan
}

In a group of patients experiencing pain during wound debridement, the effects of topical lidocaine gel were compared with the effects of a placebo gel in reducing pain and completing debridement. Ten adult subjects with painful wounds having a surface area less than $300 \mathrm{sq} . \mathrm{cm}$., and requiring hydro therapy and debridement, were each evaluated for pain during four sequential treatment episodes. Using a randomized, double-blind protocol, the hospital pharmacy provided, in numbered sequence, $10 \mathrm{ml}$. containers of either plain gel or $2 \%$ lidocaine gel. The gel was applied to the wound following hydro therapy and the wound debrided ten minutes later. After the wound was dressed, the subject and the nurse independently rated the level of pain experienced by the subject, and the nurse rated the percentage of debridement accomplished.

Significantly lower pain levels were reported (Subject: $T=2.24(7), p=.06$ and Nurse: $T=2.46(7), p=.04)$ and more complete debridement accomplished $(T=2.35(7), p=.05)$ during treatments with lidocaine than with plain gel. No adverse effects were noted from either gel.

Supported in part by a Biomedical Research Grant. 


\title{
Ethics of Clinical Intervention Studies
}

\author{
Discussant \\ Anne J. Davis, Ph.D. \\ Professor \\ Mental Health \& Community Nursing \\ University of California, San Francisco \\ San Francisco, California
}

This paper raise the question as to whether it is possible to simultaneously maintain our deeply held values of human rights and the advancement of science. This in turn raises questions as to our understanding of what it means to be human, the concept of humanhood. Humans have human rights and rights imply obligations. One fundamental ethical tension is that between individual rights and the common good. Selected aspects of ethics and clinical intervention studies were considered. The major point is made that it is essential to examine the ethical principles underlying the norms established in various codes and regulations and to apply them in specific situations. Six research norms were identified: good research design, balance of harm and benefit, competence of the investigators, informed consent, equitable selection of subjects, compensation for research-related injury. Four ethical principles underlie these norms: beneficence, nonmaleficence, respect for persons, and justice. There is no universal agreement as to the interpretation or weighting of these ethical principles. This author takes the stance that concerns for beneficence should not outweigh the principle of respect for persons. 